\title{
Study of Energy Absorption Efficiency for a Few Thin-walled Tubes in Axial Crushing*
}

\author{
Tsutomu UMEDA $^{* *}$, Koji MIMURA ${ }^{* *}$ and Takahiro MORISAKA ${ }^{* * *}$ \\ ** Division of Mechanical Engineering, Graduate School of Engineering, Osaka Prefecture University \\ 1-1 Gakuen-cho, Naka-ku, Sakai, Osaka 599-8531, Japan \\ *** Graduate School of Engineering, Osaka Prefecture University \\ 1-1 Gakuen-cho, Naka-ku, Sakai, Osaka 599-8531, Japan \\ E-mail: umeda@me.osakafu-u.ac.jp
}

\begin{abstract}
With a view to examine the axial crushing behavior of thin-walled structures, which are used as energy-absorbing components of transports under various dynamic compressive loads, some thin-walled circular and regular polygonal tubes were investigated mainly by the FEM simulation. The former examination was focused on the effects of the reinforcement with circular ribs placed at regular intervals in the axial direction and the moderation of load undulations due to corrugated pattern on the load-displacement relation. On the other hand, the latter examination was focused on the effects of the shape of cross section (the number of apexes and the thickness). The absorbed energy per unit volume and some indexes of efficiency were used for the examination of those axially crushing tubes. Especially, the effective range of the interval between ribs, the aspect ratio of rib, the dimensions of corrugation, and the number of apexes were primarily discussed from the view point of the comprehensive efficiency which laid emphasis on the absorbed energy per unit volume.
\end{abstract}

Key words : Thin-walled Tube, Dynamic Progressive Buckling, Energy Absorption

\section{Introduction}

Remarkable developments of transports in speed and diffusion have caused severe and many-faceted requirements for the reliability of the machinery under high-speed working conditions. The requirements for safety and environmental issues have grown increasingly and cause the advancement of the shock-absorber's performance on energy-absorbing efficiency and weight reduction. The progressive buckling of the thin-walled circular tube has been stud$\operatorname{ied}^{(1)-(10)}$ for the understanding of the crushing behavior of shock-absorbing components. The behavior of the altered thin-walled circular tube with ribs $^{(11)-(13)}$, grooves ${ }^{(14),(15)}$, corrugated pattern $^{(16)-(19)}$ or foam filler ${ }^{(20)-(22)}$ has also been studied. Furthermore, the axial crushing behavior of the square tubes (plain one ${ }^{(23),(24)}$ and reinforced ones with ribs, foam filler, and so on ${ }^{(25),(26)}$ ) and some other regular polygonal tubes ${ }^{(27),(28)}$ has been studied, too.

In this paper, some thin-walled tubes subjected to dynamic axial compression were investigated to contribute to the determination of design policy mainly by the FEM simulation using the explicit code LS-DYNA ${ }^{(29)}$ Ver.960. First, the circular tube with some circular ribs placed at regular intervals in the axial direction was modeled with the hexahedral solid element with 8 nodes, and its axially crushing behavior was calculated with varying the interval between ribs or the dimensions of rib to evaluate the effects of ribs on the energy absorption efficiency. Second, the axially corrugated circular tube was modeled with the 3D-shell element with 4 nodes to evaluate the effects of the corrugated pattern on the moderation of load undulations on the load-displacement relation. Finally, the regular polygonal tubes were also treated to evaluate the effects of the shape of cross section (the number of apexes and the thickness) on the energy absorption efficiency and the buckling mode. In order to evaluate the performance

${ }^{*}$ Received 19 Apr., 2010 (No. 10-0169) [DOI: 10.1299/jmmp.4.875]

Copyright @ 2010 by JSME 
of thin-walled tubes, the absorbed energy per unit volume was evaluated together with some indexes of efficiency through the simulations.

\section{Indexes of performance of shock absorber}

So far, some indexes for the evaluation of the shock absorber's performance have been $\operatorname{proposed}^{(3),(7),(9)}$. The shock absorbing component should have high energy absorption efficiency, the flatness of the resultant load time history to avoid excessive deceleration, and high compressibility to preserve the space for the human without increasing the mass. In this study, the absorbed energy per unit volume $e_{V}$, the overshoot of the load $\eta_{O}$, the coefficient of variation of the load $\eta_{V}$ and the geometric efficiency (the volumetric efficiency, the stroke efficiency) $\eta_{G}$ are defined to evaluate the performance of the thin-walled tubes, as follows:

$$
\left\{\begin{array}{l}
e_{V}=E_{e} / V_{e}, \\
\eta_{O}=\frac{P_{\max }-\bar{P}_{e}}{\bar{P}_{e}}, \\
\eta_{V}=\frac{a_{e}}{\bar{P}_{e}} \text { with } a_{e}=\sqrt{1 / N \Sigma_{i=1}^{N}\left(\bar{P}_{e}-P_{i}\right)^{2}}, \\
\eta_{G}=\frac{\Delta L_{\lim }}{L},
\end{array}\right.
$$

where $E_{e}$ and $V_{e}$ are the absorbed energy up to the displacement $\Delta L_{e}$ and the crushed-part volume at $\Delta L_{e}$ respectively. $P_{\max }$ and $\bar{P}_{e}$ are the maximum load and the mean load up to $\Delta L_{e}$ (see Fig.1). $P_{i}$ is the $i$-th discrete value of the load $P$, which is obtained from the time history of $P$ by sampling with the time interval $t_{S}$, and $N$ is the sampling number up to $\Delta L_{e} . L$ is the initial length of the shock absorber in the axial direction, and $\Delta L_{\text {lim }}$ is the displacement where the load abruptly increases under the densification condition.

$e_{V}$ is similar to the absorbed energy per unit mass and the mean axial stress. In this study, the crushed part is defined as the domain where the equivalent plastic strain exceeds $6 \%$ of its maximum value ${ }^{(10)}$. The definition of $\eta_{O}$ is a little different from that in the transient response and is affected by $\Delta L_{e} . P_{\max }$ is defined as the first peak load because the violent fluctuation at the rising part of the load time history is most important for the safety at the crash, while $P_{\max }$ is sometimes defined as the second or third peak load for the real shock absorber because the first peak load is decreased by giving some initial imperfection intentionally ${ }^{(9)} \cdot a_{e}$ corresponds to the standard deviation in the statistics and depends on $t_{S} . \eta_{V}$ means the relative dispersion of $P_{i}$ and also depends on $\Delta L_{e}$. In both the experiment and the numerical simulation, the stroke $\Delta L_{e}$ should be large enough to properly evaluate $\bar{P}_{e}$ and $a_{e}$, while $t_{S}$ should be small enough for the same reason.

\section{Numerical analysis}

\subsection{Material model}

The target materials employed in this study are STKM12A (JIS G 3445: Carbon steel tubes for machine structural purposes), SPCC (JIS G 3141: Cold-reduced carbon steel sheets and strips), and annealed S20C (JIS G 4051: Carbon steel for machine structural use). Those are modeled as isotropic elastic/viscoplastic materials based on the von Mises yield criterion. The dynamic yield stress or the flow stress $\sigma_{y}$ is represented in the form of the Johnson-Cook

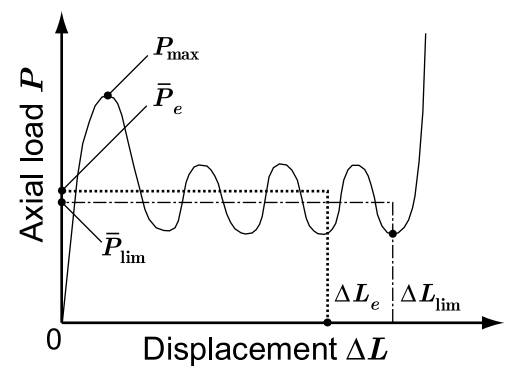

Fig. 1 Schematic of load-displacement relation 
model without thermal coupling ${ }^{(30)}$ :

$$
\sigma_{y}=\left\{\sigma_{0}+B\left(\varepsilon_{e q}^{p}\right)^{n_{J}}\right\}\left(1+C \ln \frac{\dot{\varepsilon}_{e q}^{p}}{\dot{\varepsilon}_{0}}\right),
$$

where $\sigma_{0}$ is the initial yield stress under the reference plastic strain rate $\dot{\varepsilon}_{0}$, and $\varepsilon_{e q}^{p}$ and $\dot{\varepsilon}_{e q}^{p}$ are the equivalent plastic strain and the equivalent plastic strain rate respectively. The concrete values of material constants for STKM12A, SPCC and S20C-A, which were determined by the quasi-static and the high-speed (the nominal strain rate $\dot{\varepsilon}_{N} \simeq 10^{3} \mathrm{~s}^{-1}$, by the non-coaxial Hopkinson bar method) tension tests, are shown in Table 1. The flow stress-plastic strain relations of those with $\dot{\varepsilon}_{0}=1$ are shown in Fig.2. In this study, the nominal strain rate $\dot{\varepsilon}_{N}$ was fixed at $0.1 \mathrm{~s}^{-1}$ to focus the discussion on the influence of geometry, and $\dot{\varepsilon}_{e q}^{p}$ will reach about $10 \mathrm{~s}^{-1}$ during the development of wrinkle. The circular tube was made of STKM12A by cutting to be a given thickness, while the polygonal tube was made of SPCC or S20C-A. SPCC tube was made by welding two bended plates together (low precision and low cost), and S20C-A tube by the electric discharge machining (high precision and high cost). The initial yield stress of STKM12A is 2 3 times as large as that of SPCC or S20C-A, and then it is difficult to compare the experimental results between the circular and the polygonal tubes.

Table 1 Material properties of STKM12A ${ }^{(10)}$, SPCC and S20C-A

\begin{tabular}{lccccccc}
\hline Material & $\rho\left(\mathrm{kg} / \mathrm{m}^{3}\right)$ & $E(\mathrm{GPa})$ & $v$ & $\sigma_{0}(\mathrm{MPa})$ & $B(\mathrm{MPa})$ & $n_{J}$ & $C$ \\
\hline STKM12A & 7860 & 208 & 0.28 & 345 & 360 & 0.11 & 0.0065 \\
SPCC $(h=0.5 \mathrm{~mm})$ & 7860 & 208 & 0.28 & 186 & 1080 & 0.65 & 0.0408 \\
S20C-A & 7860 & 205 & 0.28 & 213 & 1028 & 0.529 & 0.0689 \\
\hline
\end{tabular}

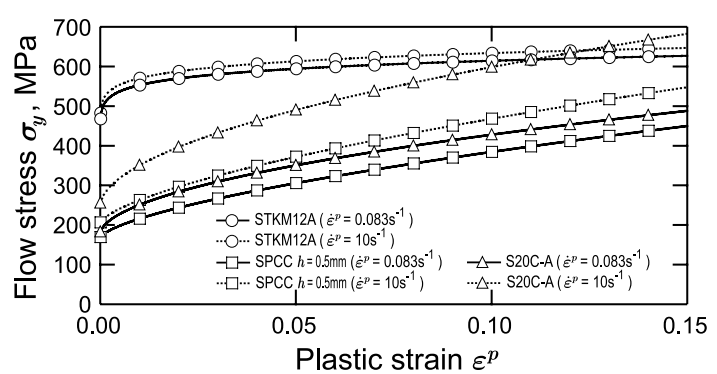

Fig. 2 Flow stress-plastic strain relations by Eq.(2) with material constants in Table 1

\subsection{Example of numerical analysis}

The experiment was conducted by the Instron universal testing machine (25T) under constant velocity condition $(500 \mathrm{~mm} / \mathrm{min})$, and then $\dot{\varepsilon}_{N}=0.083 \mathrm{~s}^{-1} \simeq 0.1 \mathrm{~s}^{-1}$ was obtained with the specimen length $L=100 \mathrm{~mm}$. On the other hand, Fig.3(a) shows an example circular tube model of the mean radius $R=17.65 \mathrm{~mm}$ and the thickness $h=0.9 \mathrm{~mm}$ with 33648 elements of the hexahedral solid type. The material is STKM12A. The velocity $V$, which rises in the shape of sine curve of the amplitude $V_{0}$ up to $50 \mu$ s and becomes constant as shown in Fig.3(a), is applied to the upper rigid block in the $-z$ direction, while the lower end of the lower elastic block is fixed in all directions. In the explicit FEM simulation, it takes enormous time to calculate relatively low speed phenomenon, so that the simulation was conducted with the conditions $V_{0}=5 \mathrm{~m} / \mathrm{s}$ and $\dot{\varepsilon}_{0}=500 \mathrm{~s}^{-1}$, which causes the strain-rate effect equivalent to $\dot{\varepsilon}_{N}=0.1 \mathrm{~s}^{-1}$. However, it must be careful with using this technique not to cause large inertial effect. The frictional force $F_{C}$ is calculated with the coefficient of friction $\mu$ in the following Coulomb's law ${ }^{(29)}$ :

$$
F_{C}=\mu N_{C}, \quad \mu=\mu_{k}+\left(\mu_{s}-\mu_{k}\right) e^{-\beta\left|V_{C}\right|},
$$

where $N_{C}$ is the contact force in the normal direction to the contact surface, and $\mu_{s}$ and $\mu_{k}$ are the static and kinetic coefficients of friction respectively. $\beta$ is the constant to vary $\mu$ continuously with the relative velocity between the contact surfaces, $V_{C}$. Table 2 shows the concrete 
values used in the simulation. Figure 3(b) shows the comparison of the load $P$-displacement $\Delta L$ relation between the experimental and calculated results. The experimental and calculated results agree well with each other, and both the crushed-tubes show the axisymmetric mode in the progressive buckling. $h / R(\simeq 0.05)$ is so large that the buckling occurs in fully plastic. In the case of relatively short circular tube, the plastic buckling load $P_{c r}^{p}$ is obtained as follows ${ }^{(1)}$ :

$$
P_{c r}^{p}=2 \pi R h \sigma_{c r}^{p}=\frac{2 \pi E_{t} h^{2}}{\sqrt{3\left(1-v^{2}\right)}},
$$

where $E_{t}$ is the tangent modulus, and $\sigma_{c r}^{p}$ is the plastic buckling stress. $E_{t}$ at the initiating buckling is evaluated to be about $19 \mathrm{GPa}$ at $\varepsilon^{p} \simeq 0.001$ by Eqs.(2) and (4).

Table 2 Properties of contact conditions for steel

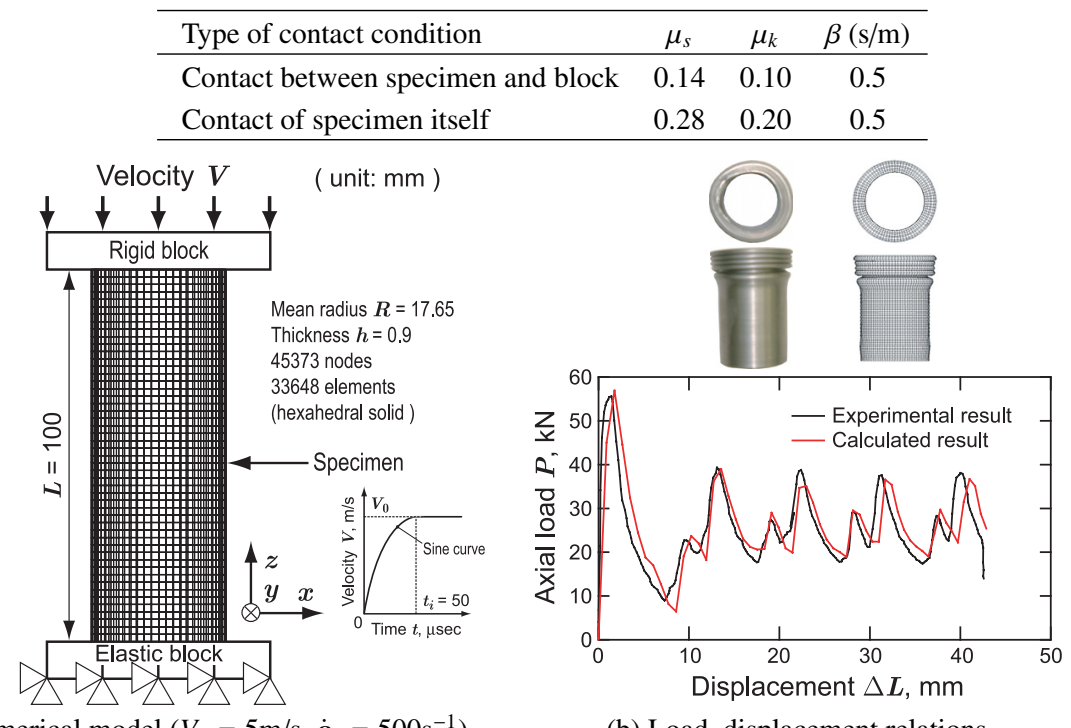

(a) Numerical model $\left(V_{0}=5 \mathrm{~m} / \mathrm{s}, \dot{\varepsilon}_{0}=500 \mathrm{~s}^{-1}\right)$

(b) Load-displacement relations

Fig. 3 Comparison between experimental and calculated results (STKM12A)

\section{Thin-walled circular tube with ribs}

The axial crushing behavior of the thin-walled circular tube greatly depends on the ratio of thickness to radius and the initial imperfection, and the tendency to crush in the nonaxisymmetric buckling mode grows with the decrease of this ratio ${ }^{(1),(2),(8)}$. The control of the buckling mode is important because $e_{V}$ in the non-axisymmetric mode is about $20 \%$ less than that in the axisymmetric one at the maximum ${ }^{(10),(12)}$. The reduction of the thickness of component is indispensable to save the machine weight, and then the reinforcing (or reducing) method of the thin-walled component is also important to keep its strength. In this section, the effect of the circular ribs (or grooves), which are placed at regular intervals in the axial direction, was investigated. This alteration will be done by cutting the circular tube on a lathe, for example. Figure 4(a) shows the model of the base plain circular tube, and Figs.4(b) and (c) show the model of the circular tube with $n_{R}$ ribs and that of the unit tube with only two ribs. $h_{R}, L_{R}$ and $L_{I}$ are the rib thickness, the rib width and the interval between two adjacent ribs. The material is STKM12A.

\subsection{Numerical examination by using whole model}

All the models are crushed up to 50mm, and the mean load $\bar{P}_{e}$ is evaluated with $\Delta L_{e}=$ 50mm. Figures 5(a) (h) show $P-\Delta L$ relations for $n_{R}=0 \sim 13$ with $L_{R}=4 \mathrm{~mm}$ and $h_{R}=2 \mathrm{~mm}$. The plain circular tube $\left(n_{R}=0\right)$ is crushed in the non-axisymmetric mode with triangular wrinkles $(h / R$ is smaller than that of the model in Fig.3(a)), while any other tube with more than 2 ribs is crushed in the axisymmetric one.

$\bar{P}_{e}$ gradually increases with $n_{R}$ while $n_{R} \leq 9$, and $\bar{P}_{e}$ for $n_{R}=13$ is about 4 times larger than that for any other case. The load fluctuation after the first peak also increases with $n_{R}$ 


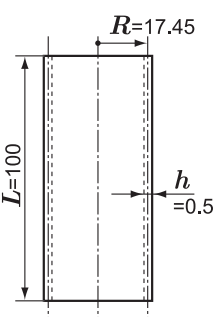

(a) Plain tube

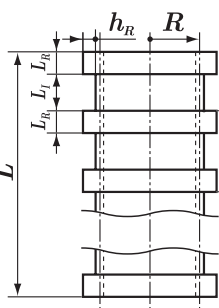

(b) With $n_{R}$ ribs
( unit: $\mathrm{mm}$ )

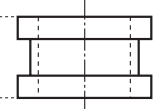

(c) Unit tube

[ Solver ]

LS-DYNA Ver. 960

hexahedral solid

Fig. 4 Models of circular tubes with $n_{R} \operatorname{ribs}\left(L_{I}=\frac{L-n_{R} L_{R}}{n_{R}-1}\right.$ in the case of (b)

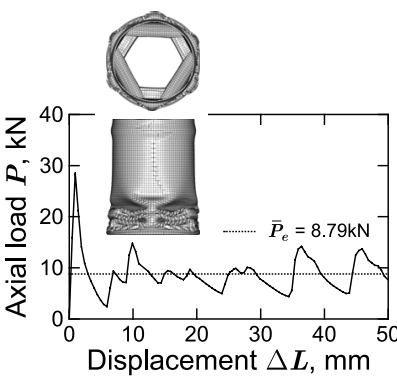

Displacement $\Delta \boldsymbol{L}$,
(a) Plain tube $\left(n_{R}=0\right)$

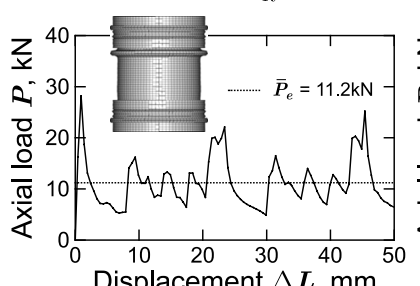

(d) $n_{R}=4$

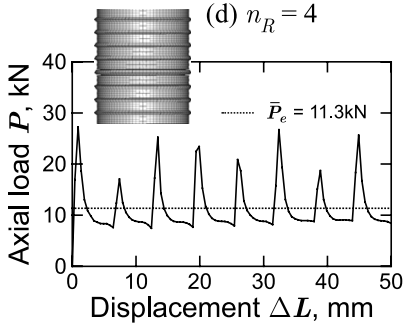

(g) $n_{R}=9$

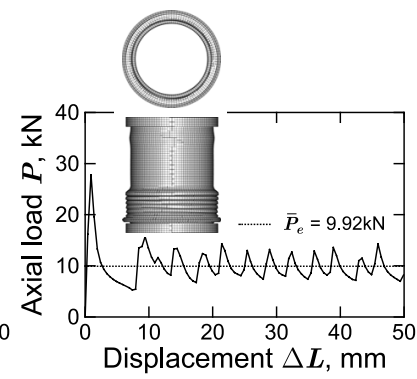

(b) $n_{R}=2$

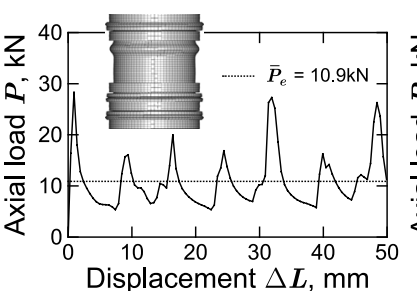

(e) $n_{R}=5$

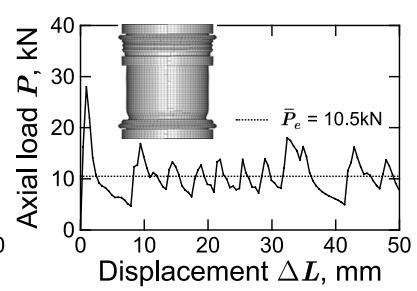

(c) $n_{R}=3$

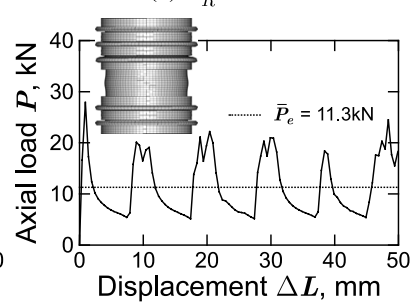

(f) $n_{R}=7$

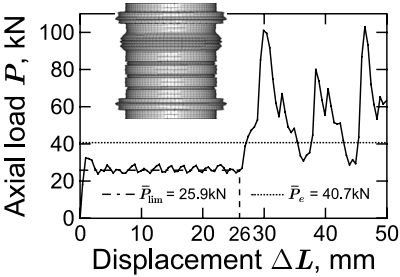

(h) $n_{R}=13\left(\Delta L_{\text {lim }} \cong 26 \mathrm{~mm}, \eta_{G} \cong 0.26\right)$

Fig. 5 Load-displacement relations $\left(L_{R}=4 \mathrm{~mm}, h_{R}=2 \mathrm{~mm}, \Delta L_{e}=50 \mathrm{~mm}\right)$

in $n_{R} \leq 9$, and only the tendency for $n_{R}=13$ is far different from that for any other case. $P$ for $n_{R}=13$ is almost constant around $26 \mathrm{kN}$ up to $\Delta L \simeq 26 \mathrm{~mm}$ but suddenly fluctuates with an enormous amplitude after that and reaches a peak value of about $101 \mathrm{kN}$. It was found by following up the calculated deformation that the original tube part of the model of $n_{R}=13$ was fully crushed until about $26 \mathrm{~mm}$, and then the rib parts also began to crush.

Figure 6 shows the formation order and the wrinkle modes of respective models. The models of $n_{R}=3 \sim 5$ yield some wrinkles in each interval $L_{I}$, and the load fluctuations of the models of $n_{R}=3$ and 4 are irregular to a greater or lesser extent, as shown in Figs.5(c) and (d) respectively. This is caused by the formation of the last wrinkle in the residual part of each interval, which is smaller than the length of one wrinkle yielded without the effect of ribs, $L_{W}$. $L_{W}$ is obtained by the classical theory of elastic buckling ${ }^{(1)}$ as follows:

$$
L_{W}=\pi\left\{\frac{2 R h}{\sqrt{3\left(1-v^{2}\right)}}\right\}^{\frac{1}{2}} \simeq 10.2 \mathrm{~mm} .
$$

$L_{I}=20 \mathrm{~mm}$ for $n_{R}=5$ yields just two wrinkles, while $L_{I}=44 \mathrm{~mm}$ for $n_{R}=3$ and $L_{I}=28 \mathrm{~mm}$ for $n_{R}=4$ yield short wrinkles lastly. The formation of wrinkle in the short region causes high resistance due to severe constraint condition at both the ends. Each of models of $n_{R}=7 \sim 13$ yields only one wrinkle in each interval, and $L_{I}$ for $n_{R}=9$ and 13 are smaller than $L_{W}$. 


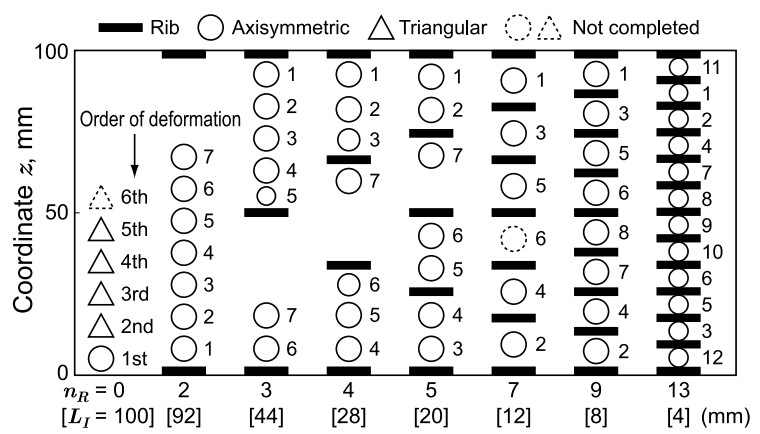

Fig. 6 Wrinkles of circular tubes with $n_{R}$ ribs $\left(L_{R}=4 \mathrm{~mm}, h_{R}=2 \mathrm{~mm}, \Delta L=50 \mathrm{~mm}\right)$

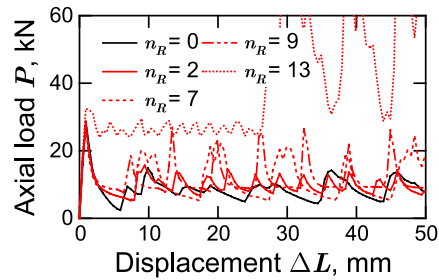

(a) Load-displacement relations

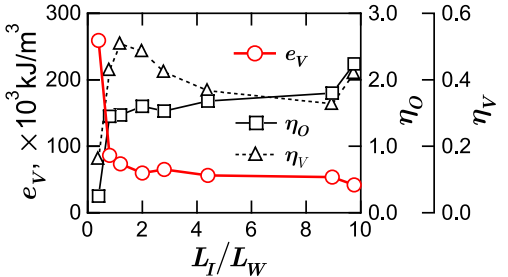

(b) $e_{V}, \eta_{O}$ and $\eta_{V}$

Fig. 7 Results of circular tubes with $n_{R}$ ribs $\left(L_{R}=4 \mathrm{~mm}, h_{R}=2 \mathrm{~mm}\right.$. $e_{V}$ is evaluated with $\Delta L_{e}=26 \mathrm{~mm}$. $\eta_{O}$ and $\eta_{V}$ only for $n_{R}=13$ are evaluated with $\Delta L_{e}=26 \mathrm{~mm}$, or else $\Delta L_{e}=50 \mathrm{~mm}$.)

Figure 7(a) shows the comparison of the $P-\Delta L$ relation among the models of $n_{R}=$ 0, 2, 7,9 and 13, and Fig.7(b) shows the changes of $e_{V}, \eta_{O}$ and $\eta_{V}$ with $L_{I} / L_{W}$. In Fig.7(a), the overall magnitude of $P$ and its fluctuation obviously increases with $n_{R}$ in $n_{R} \leq 9$, and the result for $n_{R}=13$ is quite different from the other results. In Fig.7(b), $\eta_{O}$ and $\eta_{V}$ in $n_{R} \leq 9$ are evaluated with $\Delta L_{e}=50 \mathrm{~mm}$, and those only for $n_{R}=13$ are evaluated with $\Delta L_{e} \simeq 26 \mathrm{~mm}$ because this practically means the upper limit of the crushable distance $\Delta L_{\mathrm{lim}}$ for $n_{R}=13$. However, $e_{V}$ is evaluated with $\Delta L_{e}=26 \mathrm{~mm}$ for all the models because $e_{V}$ changes with $\Delta L_{e}$ sensitively for a small number of wrinkles. In Fig.7(b), $e_{V}$ increases with decreasing $L_{I} / L_{W}$ or with $n_{R}$; the ratio of increase to the case of plain tube becomes about $28 \%$ at $L_{I} / L_{W}=9.04\left(n_{R}=2\right)$ and $520 \%$ at $L_{I} / L_{W}=0.39\left(n_{R}=13\right)$, and $\eta_{O}$ decreases with $L_{I} / L_{W}$; the ratio of decrease to the plain tube becomes about $20 \%$ at $L_{I} / L_{W}=9.04$ and $89 \%$ at $L_{I} / L_{W}=0.39$. $\eta_{V}$ also drops about $22 \%$ while $L_{I} / L_{W}$ changing from 9.83 to $9.04\left(n_{R}: 0 \rightarrow 2\right)$, but it increases about $21 \%$ as compared with the plain tube while $L_{I} / L_{W}$ changing from 9.04 to $1.18\left(n_{R}: 2 \rightarrow 7\right)$, and again it steeply decreases with $L_{I} / L_{W}$ (finally decreases about $61 \%$ ).

For the shock-absorbing component, $e_{V}$ is required to be large, and $\eta_{O}$ and $\eta_{V}$ to be small. The alteration of $n_{R}$ from 0 to 2 improves all the indexes mentioned in Section 2 except $\eta_{G}$, which is required to be large but is hardly capable of estimation with the whole model ( $\eta_{G} \simeq 0.26$ only for $n_{R}=13$ in Fig.5(h)). Then, the increase of $n_{R}$ in $2 \leq n_{R} \leq 7$ improves $e_{V}$ and $\eta_{O}$ but disimproves $\eta_{V}$. Especially, $\eta_{V}$ is the most disimproved at $n_{R}=7\left(L_{I} / L_{W} \simeq 1.18\right)$. Again, all the indexes except $\eta_{G}$ are improved in $n_{R} \geq 7\left(L_{I} / L_{W} \leq 1.18\right)$ with increasing $n_{R}$. If $\eta_{V}$ and $\eta_{G}$ can be disregarded, the increase of $n_{R}$ is always effective. If $\eta_{V}$ is the most important factor, however, $n_{R}$ should be 2 (only changing the non-axisymmetric mode to the axisymmetric one) or a certain number larger than 9 in this examination. On the other hand, the increase of $n_{R}$ obviously disimproves $\eta_{G}$.

\subsection{Numerical examination with using unit model}

Here, the model of the unit circular tube with only two ribs, which corresponds to one span of the whole circular tube with $n_{R}$ ribs as shown in Figs.4(b) and (c) $(R=17.45 \mathrm{~mm}$, $h=0.5 \mathrm{~mm}, L_{R}=4 \mathrm{~mm}, h_{R}=2 \mathrm{~mm}$ ), was treated to discuss the effects of $L_{I} / L_{W}$ on the energy absorbing efficiency. In Fig.4(c), the rib part is assumed to hardly deform as compared with the tube part, and at both the end surfaces the coefficient of friction $\mu$ is taken to be 0 . As 


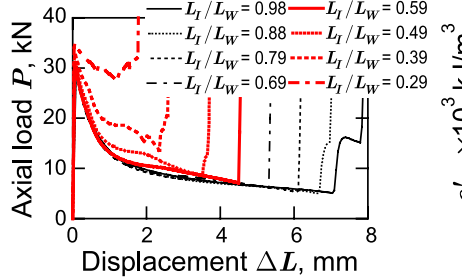

(a) Load-displacement relations

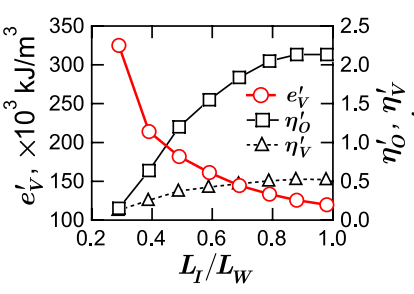

(b) $e_{V}^{\prime}, \eta_{O}^{\prime}$ and $\eta_{V}^{\prime}$

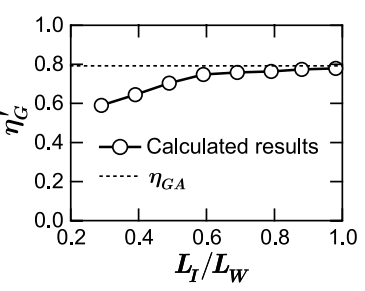

(c) $\eta_{G}^{\prime}-L_{I} / L_{W}$ relation

Fig. 8 Results of unit tubes $\left(R=17.45 \mathrm{~mm}, h=0.5 \mathrm{~mm}, L_{R}=4 \mathrm{~mm}, h_{R}=2 \mathrm{~mm}\right)$

mentioned in the subsection $4.1, \eta_{V}$ has the peak value at $L_{I} / L_{W} \simeq 1.18(\simeq 1)$ in Fig.7(b), and the secondary and following peaks of $P$ in Fig.5(f) or (g) show generally the same level close to that at the first peak. Those figures imply that the buckling behavior of all the succeedingly crushing spans will approach to that of the first crushed one as $L_{I} / L_{W}$ decreases in $L_{I} / L_{W} \leq 1$. The calculation of the whole model with small ribs costs too many computer resources, and calculating one span is enough to study the effects of $L_{I} / L_{W}$ in $L_{I} / L_{W} \leq 1$.

Figure 8(a) shows $P-\Delta L$ relations at some $L_{I} / L_{W}$ in $L_{I} / L_{W} \leq 1$ ( $L_{I}$ changes from $3 \mathrm{~mm}$ to $10 \mathrm{~mm}$ at intervals of $1 \mathrm{~mm}$ ), and the peak value of $P$ rises about $19 \%$ as $L_{I} / L_{W}$ decreases. $\Delta L_{\text {lim }}$ decreases from $7.04 \mathrm{~mm}$ to $1.77 \mathrm{~mm}$ together with $L_{I} / L_{W}$. Meanwhile, the elastic buckling load increases and approaches to that calculated for the Euler buckling of elemental $\operatorname{strip}^{(1)}$ :

$$
P_{c r}^{e}=2 \pi R\left(\frac{\pi^{2} D}{L_{I}^{2}}\right)=\frac{\pi^{3} E R h^{3}}{6\left(1-v^{2}\right) L_{I}^{2}} \quad \text { with } \quad D=\frac{E h^{3}}{12\left(1-v^{2}\right)},
$$

where $D$ is the flexural rigidity of shell. $P_{c r}^{e}$ changes from $25.4 \mathrm{kN}\left(L_{I}=10 \mathrm{~mm}\right)$ to $283 \mathrm{kN}$ $\left(L_{I}=3 \mathrm{~mm}\right)$, while the simulated buckling load changes from $29.2 \mathrm{kN}$ to $34.8 \mathrm{kN}$. From Eq.(2), the load at the initial yield becomes $\sigma_{y} \times 2 \pi R h \simeq 26.6 \mathrm{kN}$ for $\varepsilon^{p}=0$ and $\dot{\varepsilon}^{p} / \dot{\varepsilon}_{0} \simeq 0.083$. Then, the buckling occurs in fully plastic region at the following load ${ }^{(1)}$ :

$$
P_{c r}^{p}= \begin{cases}\frac{\pi^{3} E_{t} R h^{3}}{6\left(1-v^{2}\right) L_{I}^{2}}=2 \pi R h \times \frac{\pi^{2} E_{t}}{12\left(1-v^{2}\right)}\left(\frac{h}{L_{I}}\right)^{2} & \text { if } L_{I} \leq L_{H} \\ \frac{2 \pi E_{t} h^{2}}{\sqrt{3\left(1-v^{2}\right)}}=2 \pi R h \times \frac{E_{t}}{\sqrt{3\left(1-v^{2}\right)}}\left(\frac{h}{R}\right) & \text { otherwise }\end{cases}
$$

$L_{H}$ is the threshold value of $L_{I}$ for the bifurcation between two buckling modes and is obtained by equating the right side of Eq.(7.1) with that of Eq.(7.2). Then,

$$
L_{H}=\frac{\pi}{2}\left\{\frac{R h}{\sqrt{3\left(1-v^{2}\right)}}\right\}^{\frac{1}{2}}=\frac{L_{W}}{2 \sqrt{2}} \simeq 3.60 \mathrm{~mm} .
$$

Thus, the plastic buckling stress $\sigma_{c r}^{p}=P_{c r}^{p} /(2 \pi R h)$ is the function of $h / L_{I}$ if $L_{I} \leq L_{H}$. In Fig.8(a), $P_{c r}^{p}$ is almost constant in $6 \mathrm{~mm} \leq L_{I} \leq 10 \mathrm{~mm}\left(0.59 \leq L_{I} / L_{W} \leq 0.98\right)$, so that $E_{t}$ is evaluated to be about $31 \mathrm{GPa}$ in that range by Eq.(7.2) and $26 \mathrm{GPa}$ for $L_{I}=3 \mathrm{~mm}\left(L_{I} / L_{W}=\right.$ 0.29) by Eq.(7.1). In Figs.8(b) and (c), another set of indexes $e_{V}^{\prime}, \eta_{O}^{\prime}, \eta_{V}^{\prime}$, and $\eta_{G}^{\prime}$ for the fully crushing of unit model are introduced as follows:

$$
e_{V}^{\prime}=\frac{E_{\mathrm{lim}}}{V_{I}}, \quad \eta_{O}^{\prime}=\frac{P_{\max }-\bar{P}_{\mathrm{lim}}}{\bar{P}_{\mathrm{lim}}}, \quad \eta_{V}^{\prime}=\frac{a_{\mathrm{lim}}}{\bar{P}_{\mathrm{lim}}} \quad \text { and } \quad \eta_{G}^{\prime}=\frac{\Delta L_{\mathrm{lim}}}{L_{I}},
$$

where $E_{\text {lim }}$ is the absorbed energy until $P$ rises abruptly, $V_{I}\left(=2 \pi R h L_{I}\right)$ is the volume of span, and $a_{\text {lim }}=\sqrt{1 / N \Sigma_{i=1}^{N}\left(\bar{P}_{\text {lim }}-P_{i}\right)^{2}}$. In $L_{I} / L_{W} \leq 1$, all these indexes except $\eta_{G}^{\prime}$ improve as $L_{I} / L_{W}$ decreases, that is, $e_{V}^{\prime}$ increases as $L_{I} / L_{W}$ decreases, and $\eta_{O}^{\prime}$ and $\eta_{V}^{\prime}$ decrease together with $L_{I} / L_{W}$. As compared with the whole model under the same condition of $L_{I} / L_{W}=0.39$, the absorbed energy per unit volume $e_{V}^{\prime}\left(\simeq 214 \times 10^{3} \mathrm{~kJ} / \mathrm{m}^{3}\right)$ is a little smaller than $e_{V}(\simeq$ $259 \times 10^{3} \mathrm{~kJ} / \mathrm{m}^{3}$ ) of the whole model. This is primarily caused by relatively rough mesh of the whole model and the imperfection of symmetric condition of the unit model. It is difficult to eliminate these problems, and especially the latter must be treated carefully. 


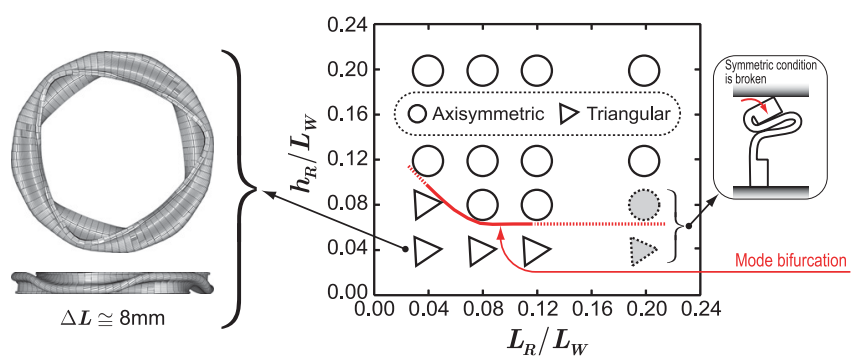

Fig. 9 Effect of $L_{R}$ and $h_{R}$ on buckling mode $\left(R=17.45 \mathrm{~mm}, h=0.5 \mathrm{~mm}, L_{I}=11 \mathrm{~mm}\right)$

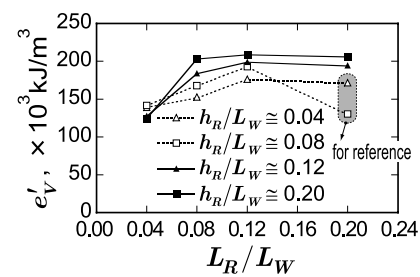

(a) $e_{V}^{\prime}-L_{R} / L_{W}$ relations

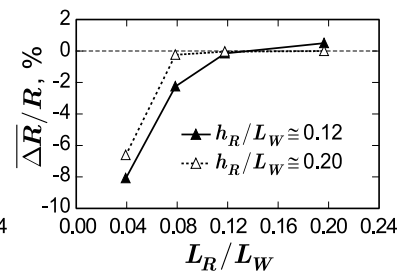

(b) $\overline{\Delta R} / R-L_{R} / L_{W}$ relations

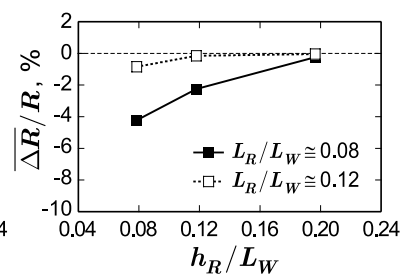

(c) $\overline{\Delta R} / R-h_{R} / L_{W}$ relations

Fig. 10 Effects of $L_{R}$ and $h_{R}$ on $e_{V}^{\prime}$ and $\overline{\Delta R} / R\left(R=17.45 \mathrm{~mm}, h=0.5 \mathrm{~mm}, L_{I}=11 \mathrm{~mm}\right)$

$\eta_{G}^{\prime}$ is required to be large, while it gradually decreases with $L_{I} / L_{W}$. In Fig.8(c), the largest value of $\eta_{G}^{\prime}(\simeq 0.78)$ is very close to the semiempirical geometric efficiency $\eta_{G A}(\simeq 0.80)$, which was proposed by Abramowicz and Jones ${ }^{(6)}$ and is given by the following equation:

$$
\eta_{G A}=1-\frac{2 x_{m}}{L_{W}^{m}}-\frac{h}{L_{W}^{p}}, \quad L_{W}^{p}=2\left(\frac{\pi R h}{\sqrt{3}}\right)^{\frac{1}{2}},
$$

where $L_{W}^{p}$ is the length of wrinkle obtained by Alexander's theory, and $L_{W} / L_{W}^{p} \simeq 1.28$ for $v=0.28 . x_{m}$ is half of the residual length of wrinkle, and Abramowicz and Jones determined $x_{m} \simeq 0.07 L_{W}^{p}$ from the observation. In Fig.8(c), the decrease of $\eta_{G}^{\prime}$ reaches about $24 \%$. On the other hand, for the whole model of $n_{R}=13\left(L_{I} / L_{W} \simeq 0.39\right), \eta_{G} \simeq 0.26$ is considerably smaller than $\eta_{G A}$, however, the net ratio of the crushed distance to the total length of tube parts except ribs is about 0.54 and is rather close to $\eta_{G}^{\prime} \simeq 0.65$. It is apparently important for $\eta_{G}$ to reduce the total length of ribs.

\subsection{Numerical examination on the geometric effects of rib (unit model)}

The geometric effects of rib are discussed by changing $L_{R}$ and $h_{R}(0.4,0.8,1.2$, and $2 \mathrm{~mm}$ respectively) with $L_{I}=11 \mathrm{~mm}$, which is long enough to yield one wrinkle. Figure 9 shows the effect of $L_{R} / L_{W}$ and $h_{R} / L_{W}$ on the buckling mode. The original circular tube crushes in the non-axisymmetric mode as shown in Fig.5(a), so that the unit model with the smallest rib of $L_{R}=h_{R}=0.4 \mathrm{~mm}\left(L_{R} / L_{W}=h_{R} / L_{W} \simeq 0.04\right)$ also crushes in the same mode. For the models of $\left(L_{R}, h_{R}\right)=(2 \mathrm{~mm}, 0.4 \mathrm{~mm}),(2 \mathrm{~mm}, 0.8 \mathrm{~mm})$, the symmetric condition with respect to the middle cross-section of rib breaks with the progress of deformation, and they crush with the behavior of extending outward. The symmetric condition for the model crushing in the non-axisymmetric mode is also not exactly realized, but even the smallest rib of $L_{R}=h_{R}=$ $0.4 \mathrm{~mm}$ hardly shows inward or outward deformation at its end surfaces until $\Delta L \simeq 3.4 \mathrm{~mm}$, and it keeps overall flatness of the surfaces until $\Delta L_{\mathrm{lim}}=8 \mathrm{~mm}$ as shown in Fig.9. Then, we compared the calculated results for all the models except those of $\left(L_{R}, h_{R}\right)=(2 \mathrm{~mm}, 0.4 \mathrm{~mm})$, ( $2 \mathrm{~mm}, 0.8 \mathrm{~mm}$ ) with each other to examine the effects of the rib geometry. The effect of $L_{R}$ on the buckling mode is very limited, and all the models of $h_{R}=0.4 \mathrm{~mm}\left(h_{R} / L_{W} \simeq 0.04\right)$ crush in the non-axisymmetric mode. However, $h_{R}$ greatly affects the buckling mode, and the model of $h_{R}=1.2 \mathrm{~mm}\left(h_{R} / L_{W} \simeq 0.12\right)$ always crushes in the axisymmetric mode, and that of $h_{R}=0.8 \mathrm{~mm}\left(h_{R} / L_{W} \simeq 0.08\right)$ also crushes in the axisymmetric mode if $L_{R} \geq 0.8 \mathrm{~mm}$.

Figures 10(a), (b) and (c) show the effects of $L_{R}$ and $h_{R}$ on $e_{V}^{\prime}$ and the average change of $R$ at the end surfaces at $\Delta L_{\mathrm{lim}}=8 \mathrm{~mm}, \overline{\Delta R}$. In Fig.10(a), $e_{V}^{\prime}$ increases together with $L_{R}$ or $h_{R}$ for all the models except those of $\left(L_{R}, h_{R}\right)=(2 \mathrm{~mm}, 0.4 \mathrm{~mm}),(2 \mathrm{~mm}, 0.8 \mathrm{~mm})$ and becomes 


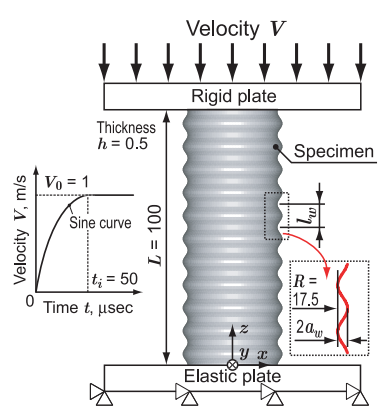

(a) Corrugated tube

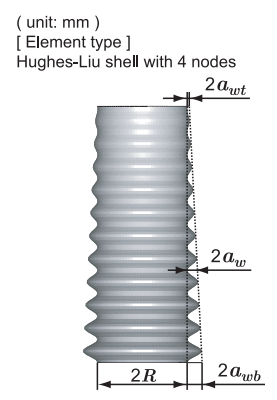

(b) Inclined corrugated tube

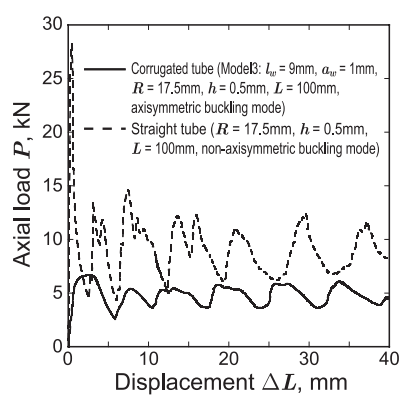

(c) Load-displacement relations

Fig. 11 Models of corrugated circular tubes $(R=17.5 \mathrm{~mm}, h=0.5 \mathrm{~mm}, L=100 \mathrm{~mm})$

almost constant in $L_{R} / L_{W} \geq 0.12$ for each $h_{R}$. In Figs.10(b) and (c), $\overline{\Delta R}$ are evaluated only for a few valid cases of the axisymmetric mode. $\overline{\Delta R} / R$ has a minus value for small $L_{R} / L_{W}$ or $h_{R} / L_{W}$ and then increases with those. Thus, the rib is deformable for small $L_{R}$ or $h_{R}$ and its rigidity increases as they increase. To insure the rigidity of rib in this examination, it should be satisfied with $L_{R} / L_{W} \geq 0.12$ and $h_{R} / L_{W} \geq 0.12$. If $n_{R}$ is large enough for the whole model,

$$
\eta_{G}=\eta_{G}^{\prime} \frac{\left(n_{R}-1\right) L_{I}}{L} \simeq \frac{\eta_{G}^{\prime}}{1+k\left(\frac{L_{W}}{L_{I}}\right)}, \quad k=\frac{L_{R}}{L_{W}} .
$$

By using $L_{I} / L_{W} \simeq 0.39, \eta_{G}^{\prime} \simeq 0.54$ (for the model of $n_{R}=13$ in the subsection 4.1 ) and $k \simeq 0.12$ (that is, $L_{R}=1.2 \mathrm{~mm}$ and $L=63.6 \mathrm{~mm}$ ), $\eta_{G}$ becomes about 0.41 , which is about $60 \%$ larger than $\eta_{G} \simeq 0.26$ for $L_{R}=4 \mathrm{~mm}$ and $L=100 \mathrm{~mm}$.

\section{Corrugated circular tube}

To improve $\eta_{O}$ and $\eta_{V}$, it is also effective to corrugate the tube. Singace and El-Sobky experimentally studied the buckling behavior of corrugated circular tubes of aluminum alloy (HT-30) and PVC ${ }^{(16)}$. They found that annealing the corrugated tube decreased the load fluctuation but also decreased the overall energy absorption. Filling the corrugated tube with the polyurethane foam enhanced the absorbed energy, while increasing the corrugation amplitude decreased it. On the other hand, the effects of the wavelength of corrugation on the buckling mode and the energy absorption were not discussed. In this section, the effects of the amplitude $a_{w}$ and the wavelength $l_{w}$ of the sinusoidal corrugation were investigated by using the 3D-shell model, which can represent the complex geometry without enlarging the total number of elements. However, $\eta_{G}$ is not treated because the 3D-shell element can't exactly reproduce the extreme deformation of complex geometry with the contact problem such as absolutely crushed tube. $a_{w}$ was varied in the narrow range of $0.6 \mathrm{~mm} \leq a_{w} \leq 1.4 \mathrm{~mm}$ to examine its effect on the buckling mode with the least reduction of absorbed energy. Figure 11(a) shows an example model of the corrugated circular tubes. The mean radius of corrugated circular tube $R_{c}(z)$ is given as follows ( $z=0$ at the bottom of tube):

$$
R_{c}(z)=R+a_{w}\left\{1-\cos \left(\frac{2 \pi}{l_{w}} z\right)\right\}
$$

where $R$ is the mean radius of the original circular tube and is $17.5 \mathrm{~mm}$. The thickness $h$ is $0.5 \mathrm{~mm}$. If $l_{w}$ is rather smaller than $L_{W}(\simeq 10.2 \mathrm{~mm}), \eta_{G}$ will disimprove in the same way as mentioned in the previous section, while larger $l_{w}$ needs large $a_{w}$, which leads to the decrease of energy absorption, to affect the buckling mode. Then, $l_{w}$ was varied around $L_{W}$ so that $R_{c}(L) \simeq R$. The simulation was conducted under the conditions $V_{0}=1 \mathrm{~m} / \mathrm{s}$ and $\dot{\varepsilon}_{0}=100 \mathrm{~s}^{-1}$, which causes the strain-rate effect equivalent to $\dot{\varepsilon}_{N}=0.1 \mathrm{~s}^{-1} . l_{w}$ and $a_{w}$ of the numerical models are shown in Table 3. Here, $e_{V}$ is evaluated with $\Delta L_{e}=40 \mathrm{~mm}$. Figure 11(c) shows the comparison of the $P-\Delta L$ relation between the corrugated and the straight tubes. Corrugating the original circular tube with $l_{w}=9 \mathrm{~mm}$ and $a_{w}=1 \mathrm{~mm}$ changes the buckling mode from non-axisymmetric to axisymmetric and considerably decreases the load fluctuation, while the absorbed energy of the corrugated tube is only about half of that of the straight one. This 
implies the importance of corrugating the tube with the smallest amplitude which is effective enough to improve $\eta_{O}$ and $\eta_{V}$.

Figure 12(a) shows the effects of $a_{w} / L_{W}$ on $e_{V}, \eta_{O}$ and $\eta_{V}$. Both $\eta_{O}$ and $\eta_{V}$ monotonically decrease (or improve) with increasing $a_{w} / L_{W}$. As $a_{w} / L_{W}$ increases, the buckling mode changes from non-axisymmetric (triangular) to axisymmetric at $a_{w} / L_{w} \simeq 0.098\left(a_{w}=1 \mathrm{~mm}\right)$. $e_{V}$ decreases with increasing $a_{w} / L_{W}$ for each mode and has the maximum value at $a_{w} / L_{w} \simeq$ 0.098 within the treated range of $a_{w}$. Figure 12(b) shows the effects of $l_{w} / L_{W}$ on $e_{V}, \eta_{O}$ and $\eta_{V}$. In this case, both $\eta_{O}$ and $\eta_{V}$ disimprove together with $l_{w} / L_{W}$. On the other hand, $e_{V}$ increases together with $l_{w} / L_{W}$ for each mode. $e_{V}$ of the axisymmetric mode is larger than that of the non-axisymmetric (elliptic) one and has the maximum value at $l_{w} / L_{W} \simeq 0.88\left(l_{w}=9 \mathrm{~mm}\right)$. The axisymmetric mode occurs within the narrow range including $0.82 \leq l_{w} / L_{W} \leq 0.88$ for $a_{w}=1 \mathrm{~mm}$.

With increasing $a_{w}$ or decreasing $l_{w}, \eta_{O}$ and $\eta_{V}$ improve but $e_{V}$ disimproves. Thus, simple corrugation is effective only for improving $\eta_{O}$ and $\eta_{V}$. Then, $e_{V}$ is tentatively improved by inclining the amplitude $a_{w}$ in the axial direction (see Fig.11(b)) as follows:

$$
a_{w}(z)=\frac{a_{w t}-a_{w b}}{L} z+a_{w b},
$$

where $a_{w t}$ and $a_{w b}$ are the amplitudes at the top and the bottom of tube. The magnitude of the bending moment at the outer edge of wrinkle (hinge line) is in proportion to the distance $2 a_{w}(z)$ under the same load. Therefore, the inclined corrugated tube starts to crush at the bottom end $\left(a_{w b} \geq a_{w t}\right.$ ), and the crushed region spreads from the bottom to the top. Figure 13(a) shows the comparison of the $P-\Delta L$ relation between the inclined corrugated (Model11) and the plain corrugated (Model3) tubes. At first, $P$ of the inclined corrugated tube is as small as that of the plain corrugated tube but increases as the crushed region goes up. The inclination is surely effective, but the non-axisymmetric mode will occur if the ratio $\left(a_{w b}-a_{w t}\right) / a_{w b}$ is too large (Model10). $e_{V}$ increases about 6\% at the maximum (Model11) as compared with Model3. Thus, the inclined corrugation is very effective in the design of the crushing behavior such as the generating order of wrinkle and the load history, and $e_{V}$ also increases a little. On the other hand, $\eta_{G}$ is affected by the geometric elements such as the radius of curvature around the hinge line and the thickness $h$ and will be approximated to $\eta_{G A}^{\prime}=1-2 x_{m} / l_{w}-h / l_{w}$ by replacing $L_{W}^{p}$ with $l_{w}$ in Eq.(10) if $l_{w} \geq L_{W}$ and the mode is axisymmetric.

\section{Regular polygonal tubes}

The axial crushing behavior of the regular polygonal tube is affected by the restraint at the ridgeline, and the number of apexes $n_{a}$ besides the transverse dimension of cross section and the thickness becomes another important parameter ${ }^{(27),(28)}$ for the buckling load of the axially crushing tube. The radius of curvature at the ridgeline moderates the stress concentration ${ }^{(27)}$ so that it also affects the behavior but was not treated here to simplify the problem. Firstly, the axial crushing behavior of SPCC tube, which was made by welding two bended plates together, was experimentally investigated. The length of side $a$ at the cross section of tube is determined so that the cross-sectional area $A$ is identical with that of the base circular tube for the specification of the thickness $h$ and $n_{a}$, as shown in Fig.14. In this experiment, the internal diameter of the base circular tube is $34.5 \mathrm{~mm}$ and $h=0.5$ or $1.2 \mathrm{~mm}(R=17.5$ or $17.85 \mathrm{~mm})$ so that $A \simeq 55$ or $135 \mathrm{~mm}^{2}$. Then, $a$ is determined by $n_{a}$ for each regular polygonal tube. The crosshead speed of the testing machine was $500 \mathrm{~mm} / \mathrm{min}$, and the testing strain rate $\dot{\varepsilon}_{N}$ was about $0.083 \mathrm{~s}^{-1}$.

Figures 15(a) (c) show the deformations of tubes of $h=1.2 \mathrm{~mm}$ at $\Delta L=70 \mathrm{~mm}$, and Figs.16(a) and (b) show $P-\Delta L$ relations and $e_{V}-n_{a}$ relation for the tubes of $h=1.2 \mathrm{~mm}$. In Fig.15(a), one can confirm the buckling mode of square tube, but in Figs.15(b) and (c) both the tubes of $n_{a}=5$ and 6 didn't yield any clear buckling mode because the accuracy of the bending process in out-of-square angle was low. The absolute error in $a$ is less than $0.05 \mathrm{~mm}$ for $n_{a}=4$ but reaches about $0.38 \mathrm{~mm}$ for $n_{a}=5$ and $0.30 \mathrm{~mm}$ for $n_{a}=6$ at the maximum. 
Table 3 Details of corrugated tubes for evaluation of energy absorption efficiency ( ${ }^{\dagger}$ straight tube: $R=17.5 \mathrm{~mm}, h=0.5 \mathrm{~mm}, L=100 \mathrm{~mm},{ }^{\ddagger}$ Model3: base model)

\begin{tabular}{lccccc}
\hline Model & $l_{w}(\mathrm{~mm})$ & $a_{w}$ or $a_{w b}(\mathrm{~mm})$ & $a_{w t}(\mathrm{~mm})$ & Buckling mode (5 wrinkles) & $e_{V}\left(\times 10^{3} \mathrm{~kJ} / \mathrm{m}^{3}\right)$ \\
\hline Straight tube & - & - & - & Triangular (all) & 134.4 \\
Model1 & 9 & 0.6 & - & Triangular (all) & 58.5 \\
Model2 & 9 & 0.8 & - & Triangular (all) & 46.4 \\
Model3 & 9 & 1 & - & Axisym. (all) & 62.6 \\
Model4 & 9 & 1.2 & - & Axisym. (all) & 56.7 \\
Model5 & 9 & 1.4 & - & Axisym. (all) & 45.6 \\
Model6 & 7.69 & 1 & - & Axisym. $\rightarrow$ elliptic (4th, 5th) & 48.7 \\
Model7 & 8.33 & 1 & - & Axisym. (all) & 60.4 \\
Model8 & 10 & 1 & - & Elliptic (all) & 51.7 \\
Model9 & 11 & 1 & - & Elliptic (all) & 58.0 \\
\hline Model10 & 9 & 1 & 0.5 & Axisym. $\rightarrow$ elliptic (5th) & 57.2 \\
Model11 & 9 & 1 & 0.6 & Axisym. (all) & 66.5 \\
Model12 & 9 & 1 & 0.7 & Axisym. (all) & 63.7 \\
Model13 & 9 & 1 & 0.8 & Axisym. (all) & 63.7 \\
Model14 & 9 & 1 & 0.9 & Axisym. (all) & 63.5 \\
\hline
\end{tabular}

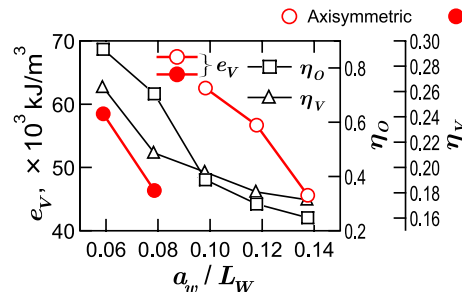

(a) $e_{V}, \eta_{O}, \eta_{V}\left(l_{w}=9 \mathrm{~mm}\right)$

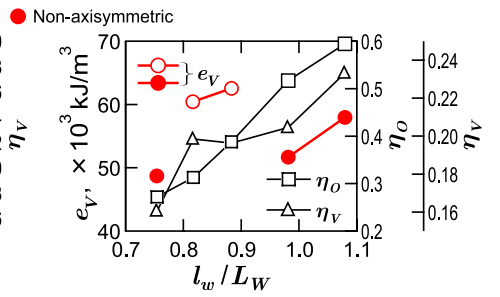

(b) $e_{V}, \eta_{O}, \eta_{V}\left(a_{w}=1 \mathrm{~mm}\right)$

Fig. 12 Results of corrugated circular tubes $\left(L_{W} \simeq 10.2 \mathrm{~mm}, \Delta L_{e}=40 \mathrm{~mm}\right)$

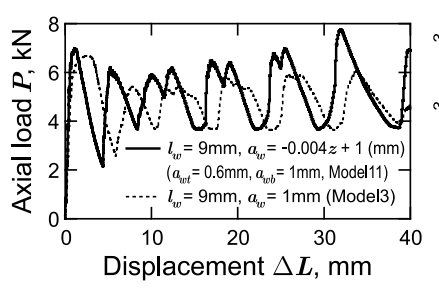

(a) Comparison with plain corrugated tube

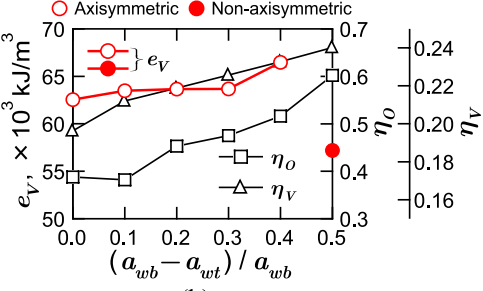

(b) $e_{V}, \eta_{O}, \eta_{V}$

Fig. 13 Results of inclined corrugated circular tubes $\left(L_{W} \simeq 10.2 \mathrm{~mm}, \Delta L_{e}=40 \mathrm{~mm}\right)$

On the other hand, $P-\Delta L$ relations in Fig.16(a) show the tendency of the overall amplitude of $P$ increasing with $n_{a}$, as reported in the previous numerical investigation ${ }^{(27)}$. Fig.16(b) also shows that of $e_{V}$ increasing with $n_{a}$. To obtain the buckling modes for $n_{a}=5$ or $6, \mathrm{~S} 25 \mathrm{C}-\mathrm{A}$ and S20C-A tubes were made with high accuracy by the electric discharge machining ('-A' means annealed material). In these cases, the absolute error in $a$ is less than $0.05 \mathrm{~mm}$ as well as that of the SPCC square tube is. Figs.15(e) and (f) show the buckling modes obtained for $n_{a}=5$ and 6 respectively. The mode of the pentagonal tube of $h=1.2 \mathrm{~mm}$ is a little broken due to the tendency of getting harder to yield the polygonal wrinkle as $h$ grows, while that of the hexagonal tube of $h=0.5 \mathrm{~mm}$ is very clear. Thus, the initial imperfection and $h$ greatly affect the buckling mode, and the polygonal tube, of which $n_{a}$ is larger than 4 , also can yield polygonal wrinkles if the process accuracy is high enough, while only the extensional mode has been reported so far. The buckling mode that emerged from this investigation can be also confirmed as one eigenmode by the eigenvalue analysis, and the pattern of polygonal wrinkle changes whether $n_{a}$ is odd or even as shown in Fig17. The side deforms inward or outward in turn along the periphery, but if $n_{a}$ is a odd number, two adjacent sides deform inward or outward in succession somewhere, as shown in the gray scale region in Fig.17. In the stacking direction of wrinkles, each side always deforms inward or outward in turn. 

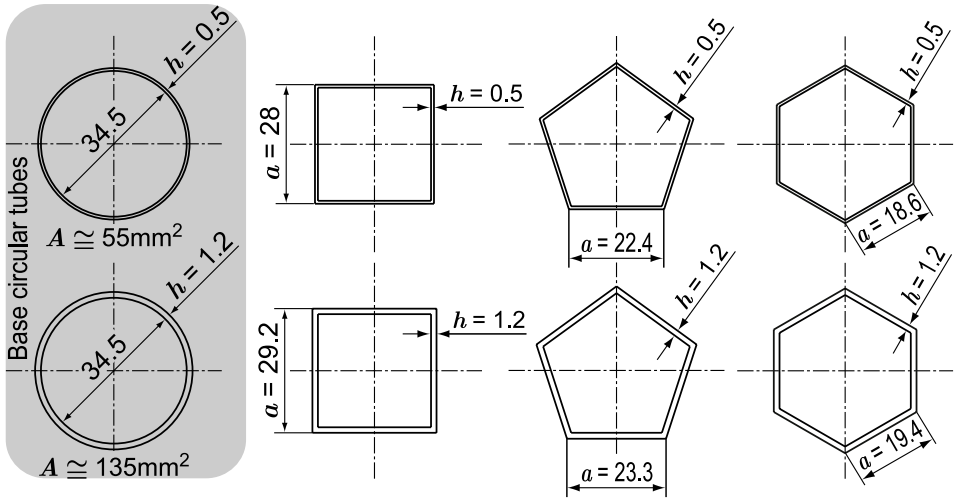

Fig. 14 Example cross sections of regular polygonal tubes (unit: $\mathrm{mm}, L=100 \mathrm{~mm}$ )

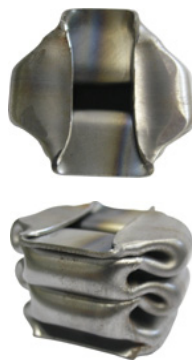

$A \cong 135 \mathrm{~mm}^{2}, \Delta L=70 \mathrm{~mm}$ (a) $n_{a}=4, h=1.2 \mathrm{~mm}$ (SPCC)

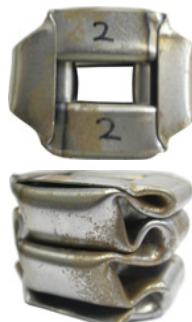

$A \cong 55 \mathrm{~mm}^{2}, \Delta L=70 \mathrm{~mm}$ (d) $n_{a}=4, h=0.5 \mathrm{~mm}$ (SPCC)
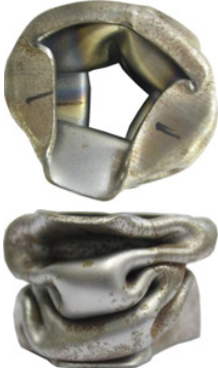

$A \cong 135 \mathrm{~mm}^{2}, \Delta L=70 \mathrm{~mm}$ (b) $n_{a}=5, h=1.2 \mathrm{~mm}$ (SPCC)

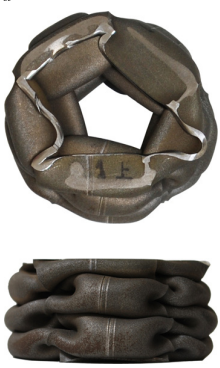

$A \cong 135 \mathrm{~mm}^{2}, \Delta L=80 \mathrm{~mm}$

(e) $n_{a}=5, h=1.2 \mathrm{~mm}(\mathrm{~S} 25 \mathrm{C}-\mathrm{A})$

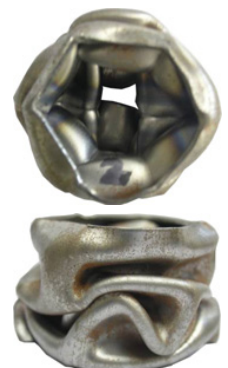

$A \cong 135 \mathrm{~mm}^{2}, \Delta L=70 \mathrm{~mm}$ (c) $n_{a}=6, h=1.2 \mathrm{~mm}$ (SPCC)

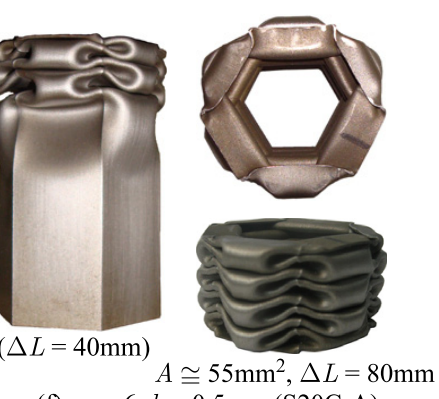

Fig. 15 Buckling modes of regular polygonal tubes $(L=100 \mathrm{~mm})$

Next, the axial crushing behavior of the regular polygonal tube was studied by the FEM simulation. Because the model without the initial imperfection causes the first wrinkle of the extensional mode only, the initial imperfection is introduced by adding the pseudo-random numbers $\Delta d_{i}\left(i=1 \sim 3 n_{n}\right)$, which are obtained by the linear congruential generator within $\left|\Delta d_{i}\right| \leq 0.04$ (unit: $\mathrm{mm}$ ), to the coordinates of $n_{n}$ nodes in the tube model. The disturbance magnitude is determined so as to be large enough to reproduce the observed buckling mode, which occurs at the least load as compared with any other possible mode, in the error range measured for the actual specimen. Figure 18(a) shows the deformed tube and $P-\Delta L$ relation calculated for the square tube, and Fig.18(b) shows the same results calculated for the hexagonal tube. Those numerical models correspond to the specimens shown in Figs.15(d) and (f) respectively. Both the calculated deformations reproduce the buckling modes obtained for the respective experiments, and the calculated $P-\Delta L$ relations also agree well with the respective experimental ones.

Figures 19(a) (g) show typical results which were calculated by varying $h / R$ and $n_{a}$. In Figs.19(a) (d), typical buckling modes of $n_{a}=7 \sim 9,12$ are shown in the view through the axial direction. The modes of $n_{a}=7$ and 8 clearly obey the patterns in Figs.17(a) and (b) respectively, while the crushing appearance of $n_{a}=9$ deviates a little from the symmetry in Fig.17(a). Furthermore, the model of $n_{a}=12$ initially shows the extensional (axisymmetric) mode and subsequently shows the triangular mode with ignoring the constraint at ridgelines. 


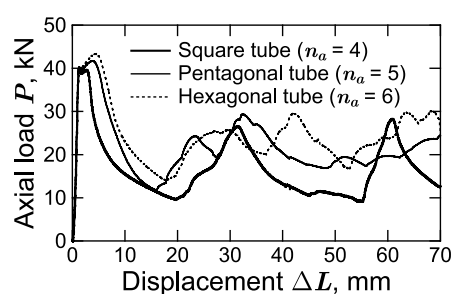

(a) Load-displacement relations

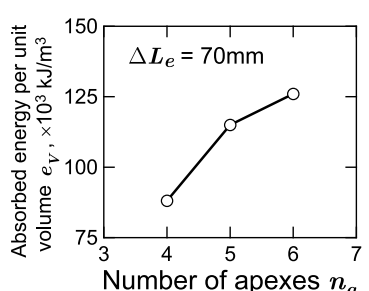

(b) $e_{V}-n_{a}$ relation

Fig. 16 Examples of experimental results (SPCC, $h=1.2 \mathrm{~mm}, A \simeq 135 \mathrm{~mm}^{2}$ )

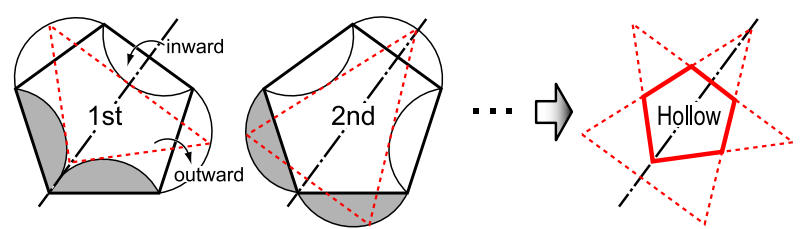

(a) Pentagonal tube $\left(n_{a}=5\right)$
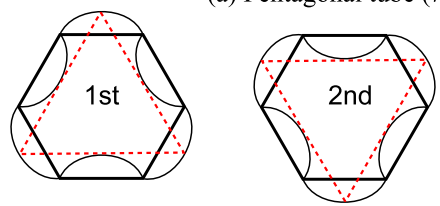

$\cdots 5$

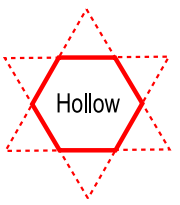

(b) Hexagonal tube $\left(n_{a}=6\right)$

Fig. 17 Schematic of polygonal wrinkles
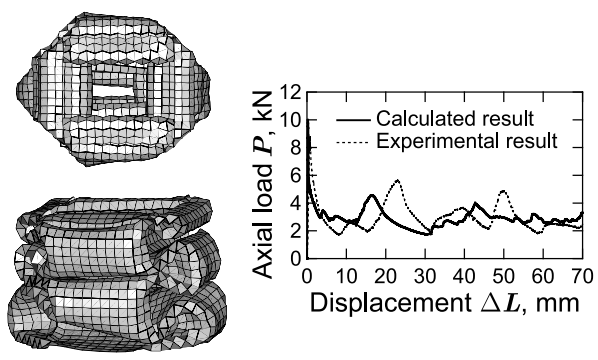

(a) $n_{a}=4, \Delta L=70 \mathrm{~mm}$ (SPCC)
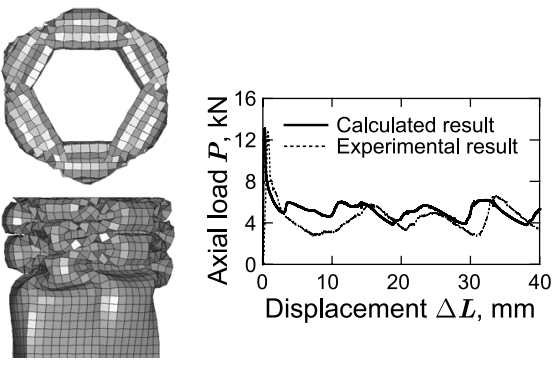

(b) $n_{a}=6$, up to $\Delta L=40 \mathrm{~mm}$ (S20C-A)

Fig. 18 Examples of calculated results $\left(h=0.5 \mathrm{~mm}, A \simeq 55 \mathrm{~mm}^{2}\right)$

The circular tube model also yields the axisymmetric mode firstly and then yields the triangular mode as shown in Fig.19(e). In Fig.19(f), the models for basic circular tubes of ( $R$, $h)=(14.25 \mathrm{~mm}, 0.5 \mathrm{~mm}),(17.5 \mathrm{~mm}, 0.5 \mathrm{~mm}),(21.75 \mathrm{~mm}, 0.5 \mathrm{~mm}),(17.7 \mathrm{~mm}, 0.9 \mathrm{~mm})$, and $(17.85 \mathrm{~mm}, 1.2 \mathrm{~mm})$ were calculated to evaluate the effects of the relative thickness $h / R$ and $n_{a}$ on $e_{V}$. The results for the basic circular tubes are also shown in the figure. $e_{V}$ increases monotonically with $h / R$. It also increases with $n_{a}$ but seems to saturate more than $n_{a}=7$. $e_{V}$ of polygonal tube is larger than that of circular tube if $n_{a} \geq 7$ or 8 . Figure $19(\mathrm{~g})$ shows the $e_{V}-n_{a}$ relation for $h / R \simeq 0.029$. In this case, $e_{V}$ has a peak at $n_{a}=9$ and then approaches the value of circular tube. $n_{a}$ for this peak is smaller than that reported in the previous study ( $n_{a}$ $=12)^{(27)}$. This difference seems to be caused by the introduce of the initial imperfection, and the obtained realistic buckling mode reduces $e_{V}$ on the whole.

\section{Conclusions}

In order to examine the effects of the circular ribs, the corrugation of tube and the regular polygonal tube (the ridgeline) on the efficiency of the energy absorbing component, a series of numerical simulations and experiments for thin-walled circular and regular polygonal tubes of some dimensions was carried out. The circular ribs, which are placed at regular intervals in the axial direction, improves the absorbed energy per unit volume $e_{V}$ and the overshoot of the load $\eta_{O}$, while the coefficient of variation of the load $\eta_{V}$ shows the complex behavior and the geometric efficiency $\eta_{G}$ always disimproves. If the interval of ribs is less than the length of one wrinkle yielded without the effect of ribs, all the indexes except $\eta_{G}$ will improve. $\eta_{G}$ 


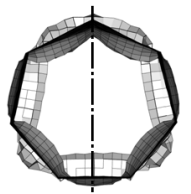

(a) $n_{a}=7, \Delta L \cong 10 \mathrm{~mm}$ $h / R \cong 0.029$

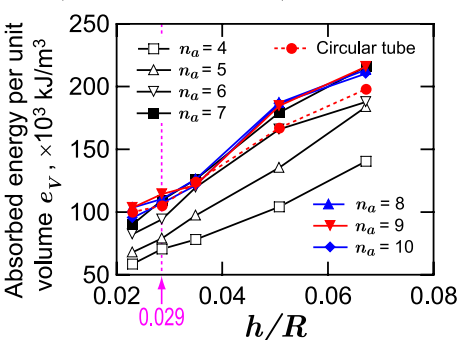

(f) Effect of $h / R$ on $e_{V}$

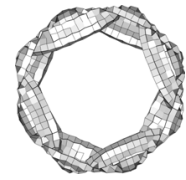

(b) $n_{a}=8, \Delta L \cong 40 \mathrm{~mm}$ $h / R \cong 0.023$

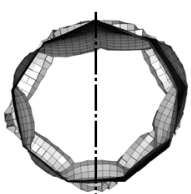

c) $n_{a}=9, \Delta L \cong 10 \mathrm{~mm}$ $h / R \cong 0.023$

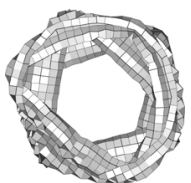

(d) $n_{a}=12, \Delta L \cong 40 \mathrm{~mm}$ $h / R \cong 0.029$

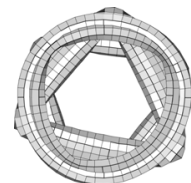

e) Circular, $\Delta L \cong 40 \mathrm{~mm}$ $h / R \cong 0.067$

Fig. 19 Typical buckling modes, $e_{V}-h / R$ and $e_{V}-n_{a}$ relations (S20C-A, $\left.\Delta L_{e}=40 \mathrm{~mm}\right)$

can be improved to some extent by reducing the dimensions of rib.

Corrugating tube can decrease the load fluctuation but also decreases the overall energy absorption. Moreover, the increase of the corrugation amplitude decreases $e_{V}$, while the increase of the corrugation period increases $e_{V}$ a little in the narrow range. The inclined corrugation is another improving method for $e_{V}$, however, the effect is not so large, too. The inclined corrugation enables to design the load history and the order of wrinkle generation.

The regular polygonal tube was also investigated to understand the axial crushing behavior of the general polygonal-tube-type shock absorber. The experiments using the specimen of high process accuracy reveals that the regular polygonal tube also crushes in the specific buckling mode. Both the overall load amplitude and $e_{V}$ increase with the number of apexes $n_{a}$. A series of numerical simulations with varying $h / R$ shows that $e_{V}$ also increases with $h / R$ ( $R$ is the mean radius of the base circular tube). $e_{V}$ of polygonal tube saturates more than $n_{a}=7$ and is larger than that of circular tube if $n_{a} \geq 7$ or 8 . In the $e_{V}-n_{a}$ relation of $h / R \simeq 0.029, e_{V}$ has a peak at $n_{a}=9$ and then approaches the value of circular tube. $n_{a}$ for this peak is smaller than that reported in the previous study $\left(n_{a}=12\right)^{(27)}$. This difference seems to be caused by the introduce of the initial imperfection, and the obtained realistic buckling mode reduces $e_{V}$ on the whole.

\section{References}

(1) Timoshenko, S.P. and Gere, J.M., Theory of Elastic Stability, (1961), pp.457-473, McGraw-Hill.

( 2 ) Horton, W.H., Bailey, S.C. and Edwards, A.M., Nonsymmetric Buckle Patterns in Progressive Plastic Buckling, Proceedings of the Society for Experimental Stress Analysis, Vol.23, No.2 (1966), pp.433-444.

( 3 ) Pugsley, Sir A., The Crumpling of Tubular Structures under Impact Conditions, Proceedings of the Symposium on The Use of Aluminum in Railway Rolling Stock, Institute of Locomotive Engineers, The Aluminum Development Association, London, (1960), pp.33-41.

( 4 ) Alexander, J.M., An Approximate Analysis of the Collapse of Thin Cylindrical Shells under Axial Loading, The Quarterly Journal of Mechanics and Applied Mathematics, Vol.13, Pt.1 (1960), pp.10-15.

( 5 ) Murase, K., Katori, H. and Nishimura, T., Viscoplastic Large Deformation Analysis by Finite Element Method and Its Applications, Bull. Japan Soc. Mech. Eng., Vol. 26 (1983), pp.923-929.

( 6 ) Abramowicz, W. and Jones, N., Dynamic Axial Crushing of Circular Tubes, Int. J. Impact Engng., Vol.2, No.3 (1984), pp.263-281. 
( 7 ) Jones, N., Structural impact, (1989), pp.385-400, Cambridge University Press.

( 8 ) Toi, Y., Discrete Limit Analysis of Steel Structures (in Japanese), (1990), pp.101-113, Baifukan.

( 9 ) Fujiwara, A. and Hashimura, T., Design Considerations Concerning Axial Collapse Characteristics of Circular Tubes, Transactions of the Japan Society of Mechanical Engineers, Series A, Vol.64, No.620 (1998), pp.1069-1076.

(10) Umeda, T., Mimura, K. and Umezaki, Y., Energy Absorption Efficiency and Mode Bifurcation of Thin-Walled Circular Tubes under Dynamic Axial Crushing, Journal of the Society of Materials Science, Vol.55, No.9 (2006), pp.799-806.

(11) Adachi, T., Takemoto, T., Araki, W. and Yamaji, A., Impact Energy Absorption of ThinWalled Cylinders with Ribs, Journal of the Society of Materials Science, Vol.53, No.3 (2004), pp.241-246.

(12) Tanimura, S., Mimura, K., Ishikawa, Y. and Umeda, T., Dynamic Axial Crushing Test to Thin-Walled Circular Tube Specimen and Evaluation of Its Energy Absorption Ability, Transactions of the Japan Society of Mechanical Engineers, Series A, Vol.65, No.635 (1999), pp.1622-1628.

(13) Kawachi, K., Umeda, T. and Mimura, K., Effect of Ribs on Energy Absorption under Axial Compression of Thin-Walled Tube, Proceedings of the 82th Kansai Branch Regular Meeting of the Japan Society of Mechanical Engineers, No.074-1 (2007), 7/21.

(14) Mamalis, A.G., Manolakos, D.E., Viegelahn, G.L., Vaxevanidis, N.M. and Johnson, W., The Inextensional Collapse of Grooved Thin-Walled Cylinders of PVC under Axial Loading, Int. J. Impact Engng., Vol.4, No.1 (1986), pp.41-56.

(15) Mamalis, A.G., Viegelahn, G.L., Manolakos, D.E. and Johnson, W., Experimental Investigation into the Axial Plastic Collapse of Steel Thin-Walled Grooved Tubes, Int. J. Impact Engng., Vol.4, No.2 (1986), pp.117-126.

(16) Singace, A.A. and El-Sobky, H., Behaviour of Axially Crushed Corrugated Tubes, Int. J. Mech. Sci., Vol.39, No.3 (1997), pp.249-268.

(17) Chen, D.H. and Hiratsuka, T., A Theoritical Analysis of Axial Crushing of Cylindrical Tubes with Corrugated Surface, Transactions of the Japan Society of Mechanical Engineers, Series A, Vol.73, No.729 (2007), pp.603-610.

(18) Chen, D.H., Hattori, K. and Ozaki, S., Axial Crushing Characteristics of Circular Tubes with Radial Corrugation, Transactions of the Japan Society of Mechanical Engineers, Series A, Vol.73, No.735 (2007), pp.1209-1216.

(19) Ohno, T., Umeda, T. and Mimura, K., Effect of Initial Surface Geometry of Thin-Walled Circular Tubes on Energy Absorption and Stability of Buckling Load, Proceedings of the 20th Computational Mechanics Conference, No.07-36 (2007), pp.237-238.

(20) Reddy, T.Y. and Wall, R.J., Axial Compression of Foam-Filled Thin-Walled Circular Tubes, Int. J. Impact Engng., Vol.7, No.2 (1988), pp.151-166.

(21) Hanssen, A.G., Langseth, M. and Hopperstad, O.S., Static and Dynamic Crushing of Circular Aluminum Extrusions with Aluminum Foam Filler, Int. J. Impact Engng., Vol.24 (2000), pp.475-507.

(22) Mimura, K., Umeda, T., Yamashita, K., Wakamori, T. and Tanimura, S., Dynamic Buckling Behavior of Foam-Filled Metal Tube, Proceedings of the 4th International Symposium on Impact Engineering, Vol.1 (2001), pp.111-116.

(23) Wierzbicki, T. and Abramowicz, W., On the Crushing Mechanics of Thin-Walled Structures, Journal of Applied Mechanics, Vol.50 (1983), pp.727-734.

(24) Abramowicz, W. and Jones, N., Dynamic Axial Crushing of Square Tubes, Int. J. Impact Engng., Vol.2, No.2 (1984), pp.179-208.

(25) Han, J. and Yamazaki, K., A Study on Maximization of Dynamic Crushing Energy Absorption of Square Tubes with and without Stiffener, Transactions of the Japan Society of Mechanical Engineers, Series A, Vol.65, No.632 (1999), pp.887-893.

(26) Hanssen, A.G., Langseth, M. and Hopperstad, O.S., Static and Dynamic Crushing of 
Square Aluminum Extrusions with Aluminum Foam Filler, Int. J. Impact Engng., Vol.24 (2000), pp.347-383.

(27) Nakazawa, Y., Tamura, K., Kusaka, T. and Hojo, M., Effects of Cross Sectional Shape on Plastic Buckling Behavior of Thin-Walled Polygonal Shell Members, Transactions of the Japan Society of Mechanical Engineers, Series A, Vol.73, No.727 (2007), pp.331337.

(28) Nakazawa, Y., Tamura, K., Kusaka, T. and Hojo, M., Effect of Material Thickness on Plastic Buckling Behavior of Thin-Walled Polygonal Shell Members, Transactions of the Japan Society of Mechanical Engineers, Series A, Vol.73, No.731 (2007), pp.828834.

(29) Livermore Software Technology Corporation, LS-DYNA Ver.960 USER'S MANUAL, (2001).

(30) Johnson, G.R. and Cook, W.H., A Constitutive Model and Data for Metals Subjected to Large Strains, High Strain Rates and High Temperatures, Proceedings of the 7th International Symposium on Ballistics, (1983), pp.541-547, Am. Def. Prep. Org. (ADPA). 